\title{
Investigating Initial Driver Intention on Overtaking on Rural Roads
}

\author{
Fei Yan, Mark Eilers, Lars Weber, Martin Baumann
}

\begin{abstract}
Driver intention recognition is essential to the development of advanced driver assistance systems providing real-time support. Current approaches for the recognition of overtaking intentions focus on drivers' observable behaviors, neglecting the fact that the intention to overtake a slower lead car emerges earlier than the resulting behavior. This paper aims to distinguish the "intention emerging process", when drivers form the initial intention to overtake, from the "action executing process", when drivers execute the overtaking maneuver. A driving simulator study has been conducted to investigate the influence of the lead vehicle type and lead vehicle speed on initiating driver' intention on overtaking on rural roads, and the effect of the complexity of the oncoming traffic on executing overtaking. The results show that the initial driver intention to overtake appears much earlier than the execution of the overtaking maneuver. The lead vehicle speed has a significant influence on initial driver intention in the "intention emerging process", while time to overtake increases with the number of the oncoming vehicles in the "action execution process". These results can contribute to the development of models for driver intention recognition by extending the prediction horizon from the recognition to a prediction of driving maneuvers.
\end{abstract}

\section{INTRODUCTION}

As announced by roadmaps of car manufactures, the development of sensor technology and vehicle automation functions will enable highly or fully automated driving according to SAE J3016 level 3-5 [1] within the next decade. Important open challenges of vehicle automation are concerned with the adaption to different traffic situations and the participation in a cooperation process with the human drivers, i.e., to design automated vehicles as "team players" [2]. The EU project AutoMate proposes the "TeamMate" concept that views driver and automation as a team, where both understand and support each other in pursuing cooperatively the safe, efficient, and comfortable driving. Following this concept, the automation should be designed to provide real-time support via the Human Machine Interface (HMI), considering the mutual capabilities and limitations of driver and automation. For this, knowledge about the intentions of both the driver and the automation involved in the cooperation is required. The driver's intention needs to be recognized by the automation to identify appropriate

*Research supported by the European Union's Horizon 2020 research program project AutoMate, GA No.: 690705.

Fei Yan is with the Human Factors Department, Ulm University, Ulm, 89081, Germany (e-mail: fei.yan@uni-ulm.de).

Mark Eilers is with the Humatects GmbH, Oldenburg, 26129, Germany, (e-mail: mark.eilers@humatects.de).

Lars Weber is with the OFFIS - Institute for Information Technology, Oldenburg, 26121, Germany (e-mail: lars.weber@offis.de).

Martin Baumann is with the Human Factors Department, Ulm University, Ulm, 89081, Germany (e-mail: martin.baumann@uni-ulm.de). information to be displayed via the HMI, a topic commonly known as driver intention recognition.

In general, driver intention recognition addresses the problem of recognizing and anticipating driving maneuvers that a driver is likely to perform in the near future. However, current models of recognizing driver intentions are based on the observable behavior of the driver, e.g., the actual steering action required for the lane change as well as accelerating to increase the speed. The problem with this approach is that the intention to overtake a slower lead car emerges earlier than the behaviors mentioned above. Furthermore, as human intentions may fail to result in behaviors, some driver intentions on overtaking may not be identified on time. For example, a driver has the intention to overtake a slowly driving truck in front, but in the end, the driver doesn't overtake due to the appearance of the oncoming traffic. It can be seen here that no corresponding behavior is not equal to "no intention to overtake", and the identification of such kind of driver intention is missing. This is particularly relevant for the overtaking maneuvers on rural roads, which are highly associated with traffic crashes [3].

Hence, this paper focuses on the initial formation of a driver's intention on overtaking on rural roads. Initial driver intention on overtaking shall be defined as "driver's first willingness to overtake the lead vehicle regardless of the possibility due to the oncoming traffic". It is expected to help to distinguish between when drivers have initial intentions to overtake and when drivers execute overtaking maneuvers, which can further contribute to the improvement of driver intention recognition models.

\section{THEORETICAL BACKGROUND AND RELATED WORK}

Intentions are defined as "conduct-controlling pro-attitudes, ones which we are disposed to retain without reconsideration" [4], which are most relevant to the perception and analytical components of the human decision cycle [5]. Humans are able to naturally recognize and share the intentions of others by using cognitive representation, symbolic communication, and social engagement. For designing adaptive automation that can support humans' decision-making process, it implies the importance of the recognition of human intentions. In the driving context, as knowledge about the current intention of a human driver provides means for adaptive warnings and early interventions, driver intention recognition becomes an increasingly important topic for the development of advanced driver assistance systems.

However, human intentions may fail to cause observable behaviors, which can be described as "intention-action gaps" [6]. In the driving context, it means that the formation of a maneuver intention will eventually but not necessarily 
immediately result in the execution of a corresponding driving maneuver, unless changed due to new information. Hence, there is a potential delay between the initial formation and the actual execution of an intention. [7] states that "the intent to perform the maneuver existed before the trajectory of the vehicle was altered and can be observed earlier". Therefore, it indicates the necessity to distinguish between when drivers have the initial intentions and when they execute the maneuvers.

For the overtaking maneuver, it is assumed that there is a two-step process from initiating driver's intention to executing overtaking maneuvers. In the first step, drivers perceive the relevant traffic information until enough information is gathered to form the initial intention of overtaking or not. We call this the "intention emerging process". In the second step, drivers will either keep following the lead vehicle when no intention to overtake is triggered in the first step, or look for the possibility to overtake by observing the oncoming traffic. As soon as a sufficiently sized gap is found, the actual overtaking maneuver will be executed. We call this second step the "action executing process".

After reviewing approaches for driver intention recognition $[8,9]$, sensor input is considered that can be classified into information about the vehicle state, the driver itself, and the situational context, which is provided by internal and external sensor systems of the vehicle. Most commonly, the vehicle state, subsuming information about the vehicle dynamics and the position and orientation of the vehicle in the road, is used to compare the observable vehicle state sequence with expected sequences [10], [11], [12], [13], [14], [15]. However, this approach has severe limitations concerning the recognition of initial driver intention, because it neglects that a maneuver needs to be initialized before it can be recognized. To overcome this limitation and to extend the predictive capabilities, more sophisticated approaches consider the inclusion of driver-based input obtained from in-vehicle camera systems to recognize characteristic preparatory measures preceding the execution of a maneuver [7], [8], [12], [16], [17]. However, driver-based input may be short-lived, as the ever-increasing introduction of automation may render driver-based input misleading. Recently, new approaches try to incorporate additional information about the situational context and especially of the traffic situation to allow an actual prediction of maneuver intentions. [18] presented a model for the recognition and prediction of lane changes based on both driver-based input and the traffic situation. To reduce complexity, they condensed the traffic situations into discrete levels of occupancy for each lane.

Besides, the prediction of driving maneuvers based on the traffic situation is also addressed by gap acceptance models in the context of decision-making. Assuming the existence of a latent critical gap where a driver is indifferently accepting or rejecting a gap in traffic, gap acceptance models attempt to determine the influence of information about a driver as well as the information available to a driver on the probability that a driver accepts an available gap [3], [19]. Unfortunately, gap acceptance models require a clear definition of a gap, which is not always obvious. Furthermore, gap acceptance models are usually realized as logistic regressions, which can be overly restrictive in more complex traffic scenarios.

Although these approaches can be used to recognize or predict driver intentions, they don't explicitly target the formation of initial driver intention on overtaking as well as the delay between the initial formation and the ultimate execution of an intention.

Several studies have investigated the influence of the traffic situation on the initial formation of overtaking intentions. [20] conducted a driving simulator experiment to investigate the influence of speed difference to the lead vehicle on the decision to overtake but did not include any opposing traffic. [3] performed a driving simulator study to investigate the impact of traffic conditions, road geometry, and driver characteristics on the decision to overtake but limited their study to two combinations of speeds for the lead and oncoming vehicles. [21] extended these studies to distributions of speeds but focused on their influence on the duration and distance of overtaking maneuvers. Recently, [19] performed a similar analysis to [3] using real-world field data, however, limited their study to a single oncoming vehicle and single target speeds for all involved vehicles.

Here, we focus on the initial formation and resulting intention-action gap of overtaking intentions on rural roads, considering the influence of the lead vehicle speed as well as lead vehicle type, and the complexity of the oncoming traffic. The results are expected to contribute to the future development of models for driver intention recognition that aim to extend the prediction horizon from the recognition to the prediction of driving maneuvers.

\section{EXPERIMENT}

In our experiment, we systematically varied relevant factors to investigate the two processes for overtaking maneuvers. To investigate the "intention emerging process", we varied both the type of the lead vehicle and the speed of the lead vehicle. To investigate the "action executing process", we varied the complexity of the oncoming traffic by changing the number of vehicles, the gap size between them and their speeds.

This experiment focuses on the distinguishing when drivers have their initial intention to overtake from when they execute overtaking. In line with this focus, the following research questions (RQ) need to be answered:

- RQ1: When does the initial driver intention on overtaking appear and how long is the delay between the formation of the initial driver intention and the execution of the overtaking maneuver?

- $\quad$ RQ2: How do lead vehicle type and lead vehicle speed influence the initial driver intention on overtaking?

- RQ3: When drivers intent to overtake, how does the complexity of the oncoming traffic affect the delay between the initial formation and the execution of the overtaking maneuver?

Based on the research questions, the corresponding hypotheses $(\mathrm{H})$ are: $\mathrm{H} 1)$ It is assumed that there is a time 
interval between the appearance of the initial driver intention and the execution of the overtaking maneuver; H2) Compared to the lead vehicle type, the lead vehicle speed will have a stronger impact on the formation of driver initial intention; H3) The time to complete overtaking maneuver will increase with the complexity of the oncoming traffic.

\section{A. Participants}

Twenty-three participants with valid German driver licenses were recruited from the University of Oldenburg, Germany. As one of them experienced motion sickness in the very beginning, the remaining data of 22 participants were used for further analysis. Participants (11 males and 11 females) had an average age of 25 years old $(\mathrm{SD}=6.1)$ and were licensed for eight years $(\mathrm{SD}=5.7)$. All participants received a compensation of 12 Euro for the 1.5-hour participation.

\section{B. Apparatus and Materials}

The used driving simulator is a fixed-based simulator platform, visualizing a maximum field of view of 150 degrees via three beamers (see Fig. 1). Two displays with a resolution of $1024 * 768$ pixels are used to simulate the left and right exterior mirror. To apply adjustable force feedback on the steering wheel, accelerator, and braking pedal, three Lexium Schneider CAN bus servo drives are used. For creating the road geometry, landscape, and traffic scenario, the simulator software SILAB 6.0 is used.

\section{Experiment Design}

The driving scenario was designed as a straight two-lane (one lane for each driving direction) German rural road track with a constant speed limit of $100 \mathrm{~km} / \mathrm{h}$ (Fig. 2). Following the proposed two-step process from initiating driver's intention to executing overtaking maneuvers, factors that potentially trigger driver intention on overtaking are manipulated in the first process, while factors affecting the executing the overtaking maneuver are varied in the second process. The details of the varied factors are summarized as follows:

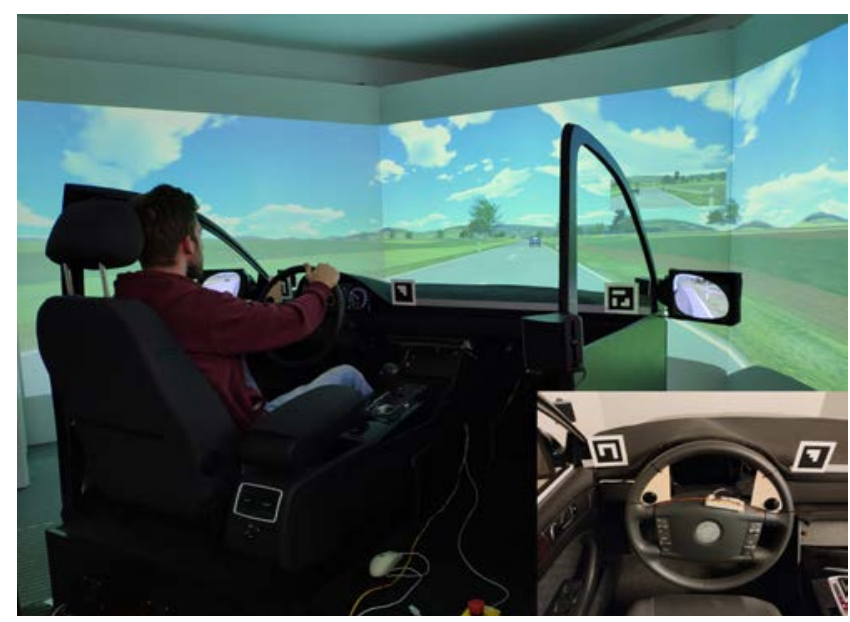

Fig. 1: Overview of the driving simulator of the University of Oldenburg. Right bottom: two buttons were installed on the steering wheel.

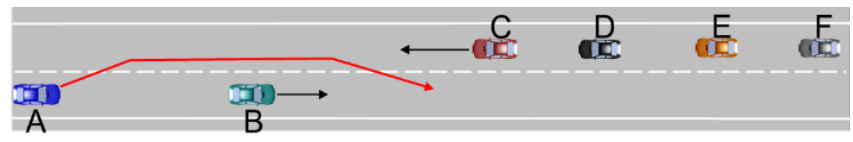

Fig. 2: The ego vehicle A follows a slower vehicle $\mathrm{B}$ on the right lane, while the oncoming traffic is varied from $0-4$ vehicles $(\mathrm{C}-\mathrm{F})$ with variable distances and speeds.

1) Lead vehicle: The lead vehicle is varied both in type and speed. Concerning the type, the lead vehicle can be a passenger car in 70 percent of the situations or a small truck (7.49 tons, $8 \mathrm{~m}$ longs) otherwise. The speed of passenger cars is varied between $65-100 \mathrm{~km} / \mathrm{h}$ with $5 \mathrm{~km} / \mathrm{h}$ intervals, the speed of trucks is either 70 or $80 \mathrm{~km} / \mathrm{h}$, held constant throughout each situation. The lead vehicle is initially set up at a distance of $180 \mathrm{~m}$ in front of the ego vehicle.

2) Complexity of the oncoming traffic: The complexity of the oncoming traffic is varied with the number of the oncoming vehicles. It consists of three groups of levels:

a) Low: No oncoming traffic.

b) Middle: A single oncoming vehicle. The distance between the ego vehicle and the oncoming vehicle is controlled via the specification of a time gap, denoted as the time to reference (TTR). TTR indicates the time (in seconds) the oncoming vehicle needs to reach a reference point. The reference point is defined as the point where the ego vehicle will reach the lead vehicle when traveling with a constant speed of $100 \mathrm{~km} / \mathrm{h}$. The speed of the oncoming vehicle is varied between $70 \mathrm{~km} / \mathrm{h}, 80 \mathrm{~km} / \mathrm{h}$, and $90 \mathrm{~km} / \mathrm{h}$ and holds constant throughout each situation, while the TTR is varied between 0-12 s, using steps of $2 \mathrm{~s}$.

c) High: Four oncoming vehicles with constant or increasing gaps between them. Their speeds are varied between $70 \mathrm{~km} / \mathrm{h}, 80 \mathrm{~km} / \mathrm{h}$, and $90 \mathrm{~km} / \mathrm{h}$, with each oncoming vehicle having the same speed and being held constant throughout a situation. The first vehicle has a TTR of 0 , while the TTR of each following oncoming vehicle was increased by 6-12 $\mathrm{s}$ to the TTR of the preceding vehicle.

In total, a set of 70 traffic situations distributed in three groups regarding the complexity of oncoming traffic (low: 10 trials; middle: 30 trials; high: 30 trials) were used.

As dependent variables, the time to have the initial driver intention on overtaking and the time to complete the overtaking maneuver are measured. To measure the initial driver intention, two black plastic buttons were installed on the left and right side of the steering wheel (see Fig.1). The participants were instructed to press the left button as soon as they intended to overtake the lead car. They had to press the right button as soon as they decided against an overtaking maneuver and follow the lead car. To measure the time until the driver executes the overtaking maneuver, we selected the point in time where the ego vehicle crossed the middle lane marking. Although this is not the earliest point in time where the steering action starts, this point could be detected accurately for all participants and trials. 
A within-subject design was used, and all participants drove 70 trials, distributed in 2 blocks, each block had 35 trials.

\section{Procedure}

After reading the handout of the instruction and filling the consent form, participants started with a training session consisting of 11 trials to get familiar with the simulator as well as the driving task. The 11 trails were selected to cover the possible overtaking situations, aiming to reduce the learning effects of the experiment.

In the beginning, participants had to accelerate to a speed of $100 \mathrm{~km} / \mathrm{h}$, which triggered the appearance of a lead vehicle $180 \mathrm{~m}$ ahead. Participants were asked to use the left or right button installed on the steering wheel to indicate their initial intentions on overtaking: Once participants had the desire to overtake the lead vehicle independent of the possibility of executing the overtaking maneuver, they should press the left button; if they did not want to overtake the lead vehicle at all, they should press the right button. After pressing the left button, participants began to assess the possibility to overtake. When possible, they executed the overtaking maneuver, and the trial ended as soon as they came back to the right lane. If participants decided against the overtaking maneuver by pressing the right button, all other vehicles disappeared immediately. In both cases, the next trial began as soon as the speed of $100 \mathrm{~km} / \mathrm{h}$ was reached.

After the training session, the first block of 35 overtaking situations was presented. After a 5-minute break, the second block followed. After these two sessions, participants were asked to fill in an online questionnaire including demographic questions and one multiple-choice question, which addresses the main reason to press the left button showing the initial overtaking intention. The given three options are: 1) the lead vehicle drives too slowly; 2) the left lane has no oncoming cars; 3 ) the lead vehicle is a truck.

In the end, they were paid 12 euros for their participation of 90 minutes.

\section{RESULT}

The data recording of the simulator ran with $60 \mathrm{~Hz}$ frequency, and it stored the simulation time (in ms), the location and speed of all vehicles and the relative bumper to bumper distances between the ego vehicle and all other vehicles. Additionally, the driver actions (steering, accelerator and brake pedal), the lateral location on the road, the use of the indicators and the button presses were recorded.

Due to the technical problems, 6 trials were missing for each of the first seven participants. Hence, we used the data of 22 participants, in total 1568 trials. Before starting the analysis, it was checked whether the collected data were valid. We removed invalid trials where participants did not follow the experimental procedure. 65 trials were excluded due to one of the following reasons: 1) both buttons were pressed;2) no button was pressed; 3) wrong buttons were pressed; 4) pressing the left button late after or during the overtaking maneuver. In the end, 1503 valid trials were analyzed in the $\mathrm{R}$ Studio software (Version 1.1.423).

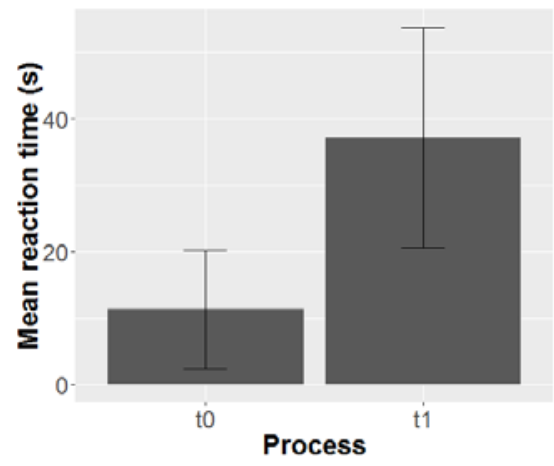

Fig. 3: The mean reaction times and standard deviations for the proposed two processes for overtaking on rural roads ( $\mathrm{t} 0$ : driver initial intention to overtake; $\mathrm{t} 1$ : the execution of the overtaking maneuver).

\section{A. Initial Driver Intention on Overtaking}

Two periods of time were measured, starting with the beginning of each trial until the driver 1) presses one of the two buttons to indicate the intention as well as 2) the point in time where the vehicle crosses the center lane marking. Fig. 3 shows the mean reaction time for the "intention emerging process" ( $\mathrm{t} 0)$ as well as the "action executing process" ( $\mathrm{t} 1)$

Fig. 3 shows the mean reaction time to form the initial driver intention and to execute the overtaking maneuver on rural roads. It can be seen that the mean reaction time of having the initial overtaking intention is $11.31 \mathrm{~s}$, while the mean reaction time until the execution of the actual overtaking action is $37.22 \mathrm{~s}$, with a delay of $25.91 \mathrm{~s}$. The result of a paired $\mathrm{t}$-Test shows that the time to have the initial overtaking intention is significantly earlier than the time to execute the overtaking maneuver $(\mathrm{t}=-44.305, \mathrm{df}=1577.6, p<0.01)$.

\section{B. The Impact of Lead Vehicle Type and Lead Vehicle Speed on Initial Driver Intention on Overtaking}

Table I shows the percentage of driver's overtaking intention categorized by the lead vehicle type. When the lead vehicle is a truck, $95 \%$ of the participants pressed the left button showing their intentions on overtaking. When the lead vehicle is a passenger car, about $64 \%$ of the participants choose the right button to show their intentions on no overtaking.

Fig. 4 illustrates the percentages of drivers' overtaking intentions influenced by the speed of the lead vehicle. In general, the slower the lead vehicle drives, the more drivers intend to overtake. Until the lead vehicle drives no faster than $80 \mathrm{~km} / \mathrm{h}$, drivers demonstrate a clear intention to overtake the lead vehicle. From $90 \mathrm{~km} / \mathrm{h}$, drivers show the strong tendency

TABLE I. THE PERCENTAGES OF THE DRIVER INTENTION ON OVERTAKING FOR THE LEAD VEHICLE AS A PASSENGER CAR AND A TRUCK

\begin{tabular}{|l|l|l|}
\hline \multirow{2}{*}{ Percentage (\%) } & \multicolumn{2}{|c|}{ Lead Vehicle Type } \\
\cline { 2 - 3 } & Passenger car & Truck \\
\hline $\begin{array}{l}\text { Overtaking } \\
\text { intention }\end{array}$ & 64 & 95 \\
\hline $\begin{array}{l}\text { No overtaking } \\
\text { intention }\end{array}$ & 36 & 5 \\
\hline
\end{tabular}




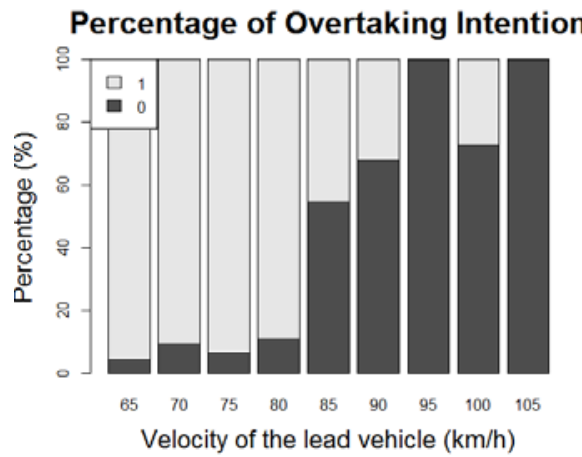

Fig. 4: The percentages of the driver's intention on overtaking for the lead vehicle as a passenger car and a truck (1: intention for overtaking; 0: intention for no overtaking).

not to overtake.

To statistically check the effect of the type and the speed of the lead vehicle on the initial driver intention on overtaking, a multiple logistic regression was performed, considering the measured driver intention as a categorical variable. In order to define the final logistic regression model, a step procedure comparing the null model with the full model was used. The null model doesn't consider any predictors, while the full model considers both the factor of the lead vehicle speed and lead vehicle type. The results show that final model includes the predictors of the lead vehicle type and also the lead vehicle speed. However, only the lead vehicle speed is the highly significant predictor to predict the driver's initial intention on overtaking $(p<.001)$, while the lead vehicle type doesn't have significant influence.

The result of the multiple-choice question about the main reason to press the left button shows that all 22 participants have chosen the first option "The lead vehicle drives too slowly".

\section{The Influence of the Complexity of the Oncoming Traffic on the Time to Overtake}

The mean times until the execution of the overtaking maneuver for the three levels of the complexity of the oncoming traffic are shown in Fig. 5. It shows that the time to overtake was the shortest in the first group with no oncoming traffic, while the overtaking time was longest for the third group with the platoon of four oncoming traffic.

The Shapiro-Wilk normality test showed that the distribution of the data of time to overtake was not normally distributed. As an alternative for ANOVA test, Kruskal-Wallis was used. The result shows that the complexity of the oncoming traffic significantly influences the time to overtake (chi-squared $=380.38, \mathrm{df}=2, p<.001$ ). As three groups have unequal numbers of observations, Nemenyi's test of multiple comparisons for independent samples was used for the post-hoc analysis, which shows that the time to overtake of each group is significantly different from the other two groups $(p<.001)$, in that the time to overtake increases with the numbers of the oncoming vehicles.

To further check the effect of the time to collision (TTC) between the oncoming vehicle and the ego vehicle in addition

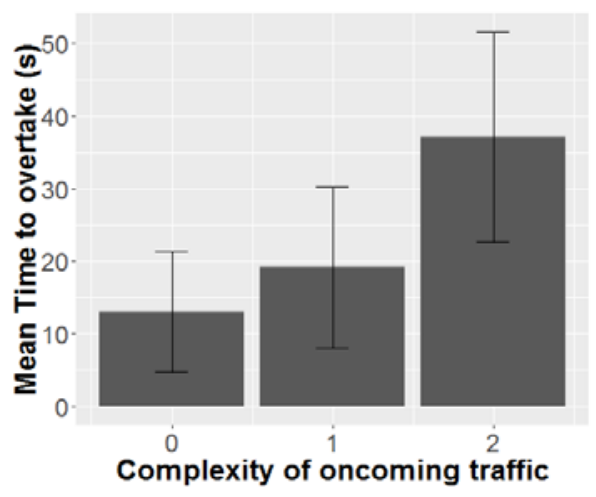

Fig. 5: The mean overtaking times and standard deviations for different complexity of oncoming traffic with standard errors ( 0 : with no oncoming traffic; 1: with one oncoming traffic; 2 : with a platoon consisting of four oncoming traffics).

to the complexity of the oncoming traffic, a multiple polynomial regression was used. The result shows that only the complexity of the oncoming traffic has a significant impact on the time to overtake with the $2^{\text {nd }}$ degree model $(p<.001)$, with a multiple R-squared of $57.76 \%$.

Lastly, the average accepted gap sizes, calculated as the gaps between the annotated beginning of the lane change and the actual lane crossing, were analyzed for the last two groups with oncoming traffic by comparing density distributions over the distance between the ego vehicle and the oncoming traffic. Participants were likely to accept the gap sizes larger than approximately $655 \mathrm{~m}$, when only a single oncoming vehicle was presented. In the presence of multiple oncoming vehicles, the average accepted gap sizes by participants were $468 \mathrm{~m}$.

\section{DISCUSSION}

Regarding the first research question (RQ1), it can be concluded that the initial driver intention on overtaking appears earlier than the execution of the overtaking, which supports the existence of the proposed two-step process of overtaking maneuvers. The significant time difference between both processes supports our assumption that it is not sufficient to rely on the occurrence of the action to make assumptions about the appearance of an overtaking intention.

With regard to the influence of the lead vehicle's speed and its type on initial driver intention on overtaking (RQ2), it can be concluded that for the "intention emerging process", only the velocity of the lead vehicle, but not the type of the lead vehicle significantly influences the formation of driver initial intention. It is also consistent with the choices of participants in the questionnaire with regard to the main reason for pressing the left button, in which all participants have chosen that the lead vehicle drives too slowly. It implies the importance of the consideration of the velocity of the lead vehicle while designing assistance systems with respect to the recognition of the initial driver intention on overtaking.

With regard to the RQ3 addressing the impact of traffic complexity on time to execute overtaking, the results show that the complexity of the oncoming traffic has a significant effect on the time required for the second process ("action executing process"). The more complex the oncoming traffic is, the 
longer drivers need to execute the overtaking maneuver. Besides, participants in the group with more oncoming vehicles accept smaller gaps for executing overtaking than the group with only one oncoming traffic, but need longer to complete overtaking. It implies that the complexity of the oncoming traffic has to be taken into account during the development of assistance systems with the function of recognizing the driver's intention.

In addition, compared to the comparative driving simulator studies investigating overtaking situations with oncoming traffic on rural roads [21], our study has extended their work by an investigation of the initial driver intention on overtaking.

However, there are still some limitations to this study. Regarding the identification of initial driver intention on overtaking, drivers may change their decisions a few times before executing the maneuver in practice. In line with this, some participants asked whether they were allowed to decide for the other button after pressing one button. They were told it was allowed if needed. The few situations where participants pressed two buttons within one trial were excluded for the analysis, as it is not the research focus of the current study. However, the change of the driver's intention still needs to be studied in future work, in order to thoroughly understand the dynamic development of driver intention on overtaking.

Last but not the least, in addition to the chosen factors that are assumed to affect initial driver intention on overtaking, other factors such as visibility, road condition, as well as driver age and gender also need to be considered for future work.

\section{CONCLUSION}

In general, the results of this study demonstrate that the initial formation of an intention to overtake appears earlier than the actual execution of the overtaking maneuver. The speed of the lead vehicle plays an essential role in the initial formation of the driver's intention of overtaking in the "intention emerging process". If an intention to overtake has emerged, the complexity of the oncoming traffic has an essential impact on the time until the overtaking maneuver is executed in the "action executing process".

Within the project AutoMate, the results of this study are currently used to develop models for driver intention recognition that extend the prediction horizon from recognition to a prediction of driving maneuvers. Especially, the results of this study are useful for designing automated vehicles with the function of recognizing driver intention on overtaking. It means that the collected empirical data can be used further to develop a model that can recognize driver intention on overtaking, with the consideration of the factors that have significantly influenced initial driver intention on overtaking on rural roads.

\section{ACKNOWLEDGMENT}

We want to thank Bertram Wortelen for his valuable comments on this paper.

\section{REFERENCES}

[1] Society of Automotive Engineers, "J3016: (R) Taxonomy and definitions for terms related to driving automation systems for on-road motor vehicles", 2016, pp.1-30.

[2] K. Christoffersen, and D. D. Woods, "How to make automated systems team players", In Advances in human performance and cognitive engineering research, Emerald Group Publishing Limited, 2002, pp. 1-12.

[3] H. Farah, S. Bekhor, A. Polus, and T. Toledo. "A Passing Gap Acceptance Model for Two-Lane Rural Highways", Transportmetrica, pp. 159-172, September 2009.

[4] M. Bratman, Intention, plans, and practical reason. Cambridge, MA Harvard University Press, 1987, vol. 10, pp.20.

[5] M. Fishbein, and I. Ajzen, Predicting and changing behavior: The reasoned action approach. New York: Psychology Press (Taylor \& Francis), 2010.

[6] N. Howard, and E. Cambria, "Intention awareness: improving upon situation awareness in human-centric environments," Human-centric Computing and Information Sciences, 2013, pp. 9.

[7] E. Ohn-Bar, A. Tawari, S. Martin, and M. M. Trivedi, "Predicting Driver Maneuvers by Learning Holistic Features," in Proc. of the IEEE Intelligent Vehicles Symposium, 2014, pp. 719-724.

[8] A. Doshi and M. M. Trivedi, "Tactical Driver Behavior Prediction and Intent Inference: A Review," In Proc. of the 14th International IEEE Conference on Intelligent Transportation Systems, 2011, pp. 1892-1897.

[9] S. Lefèvre, D. Vasquez, and Ch. Laugier, "A Survey on Motion Prediction and Risk Assessment for Intelligent Vehicles," Robomech Journal, 2014, pp. 1-14.

[10] P. Kumar, M. Perrollaz, S. Lefèvre, and C. Laugier, "Learning-based approach for online lane change intention prediction," in Proc. of the 2013 IEEE Intelligent Vehicles Symposium, 2013, pp. 797-802.

[11] A. Liu and A. Pentland, "Towards real-time recognition of driver intentions," in Proc. of the International Conference on Intelligent Transportation Systems (ITSC), 1997, pp. 236-241.

[12] N. Oliver and A. Pentland, "Driver Behavior Recognition and Prediction in a SmartCar," in Proc. of the SPIE, 2000, vol.4023, pp. 280-290,

[13] K. Torkkola, S. Venkatesan, and H. Liu. "Sensor Sequence Modeling for Driving", in Proc. of the FLARS Conference, 2005, pp. 721-727.

[14] L. Bi, C. Wang, X. Yang, M. Wang, and Y. Lie. "Detecting Driver Normal and Emergency Lane-Changing Intentions with Queuing Network-Based Driver Models", International Journal of Human-Computer Interaction, 2015, pp. 139-145.

[15] M. Liebner, M. Baumann, F. Klanner, and Ch. Stiller, "Driver Intent Inference at Urban Intersections using the Intelligent Driver Model", in Proc. of the 2012 IEEE Intelligent Vehicles Symposium, 2012, pp. 1162-1167.

[16] A. Doshi, B. T. Morris, and M. M. Trivedi, "On-Road Prediction of Driver's Intent with Multimodal Sensory Cues", Automotive Pervasive Computing, 2011, pp. 22-24.

[17] A. Jain, H. S. Koppula, B. Raghavan, S. Soh, and A. Saxena, "Car that Knows Before You Do: Anticipating Maneuvers via Learning Temporal Driving Models", in Proc. of the International Conference on Computer Vision, 2015, pp. 3182-3190.

[18] V. Leonhardt, T. Pech, and G. Wanielik, "Fusion of Driver Behaviour Analysis amd Situation Assessment for Probabilistic Manoeuvre Prediction," in UR:BAN Human Factors in Traffic, Wiesbaden, Springer Fachmedien, 2018, pp.223-244.

[19] U. Hassein, M. Diachuk, and S. Easa, "Evaluation of dynamic passing gap acceptance on two-lane highways using field data," Canadian Journal of Civil Engineering, 2017, pp.871-880.

[20] H. Farah, "Modeling drivers' passing duration and distance in a virtual environment", International Association of Traffic and Safety Sciences. Elsevier Ltd. Research, 2013, vol.37, pp. 61-67.

[21] R. Gray and D. Regan, "Perceptual processes used by drivers during overtaking in a driving simulator," Human Factors, 2005, vol.47, pp. $394-417$. 